Asian J. Med. Biol. Res. 2021, 7 (2), 214-221; doi: 10.3329/ajmbr.v7i2.55001

\author{
Asian Journal of \\ Medical and Biological Research \\ ISSN 2411-4472 (Print) 2412-5571 (Online) \\ www.ebupress.com/journal/ajmbr
}

\title{
Article \\ Genetic diversity analysis of advanced rice lines for salt tolerance using SSR markers
}

Tanoy Singha ${ }^{1 \#}$, Md. Asif Mahamud ${ }^{2 \#}$, Shahin Imran $^{3 *}$, Newton Chandra Paul ${ }^{3}$, Md. Najmol Hoque ${ }^{4}$, Tusher Chakrobarty $^{5}$, Md. Asadulla Al Galib ${ }^{6}$ and Lutful Hassan ${ }^{1}$

${ }^{1}$ Department of Genetics and Plant Breeding, Bangladesh Agricultural University (BAU), Mymensingh-2202, Bangladesh

${ }^{2}$ Department of Agricultural Chemistry, Khulna Agricultural University (KAU), Khulna-9100, Bangladesh

${ }^{3}$ Department of Agronomy, Khulna Agricultural University (KAU), Khulna-9100, Bangladesh

${ }^{4}$ Department of Biochemistry and Molecular Biology, Khulna Agricultural University (KAU), Khulna-9100, Bangladesh

${ }^{5}$ Rice Farming Systems (RFS) division, Bangladesh Rice Research Institute (BRRI), Gazipur-1701, Bangladesh

${ }^{6}$ Agronomy division, Bangladesh Rice Research Institute (BRRI), Gazipur-1701, Bangladesh

\#Tanoy Singha and Md. Asif Mahamud contributed equally to this work

*Corresponding author: Shahin Imran, Department of Agronomy, Khulna Agricultural University (KAU), Khulna-9100, Bangladesh. Phone: +8801520100207; E-mail: shahin.imran@kau.edu.bd

Received: 27 May 2021/Accepted: 28 June 2021/ Published: 30 June 2021

\begin{abstract}
Twenty-two rice lines were used to evaluate salt tolerance at the Laboratory of Biotechnology Division of Bangladesh Institute of Nuclear Agriculture (BINA), Mymensingh. Four SSR markers viz. AP3206f, RM1287, RM7075, and RM10793 were used to screen the germplasm for salt tolerance. SSR analysis revealed that the number of alleles per locus ranged from 3 to 5 with an average of 4.25 alleles per locus. Polymorphism Information Content (PIC) values ranged from 0.4762 (RM7075) to 0.7524 (AP3206f) with an average of 0.61 per locus. The highest genetic diversity (0.7810) was observed in loci AP3206f, and the lowest genetic diversity (0.5620) was observed in loci RM7075 with a mean diversity of 0.6663 . The genotypes with genetic similarity clustered together in the dendrogram based on UPGMA method and we observed seven major clusters where cluster I contained most of the genotypes. Cluster I, II, III, IV, V, VI, and VII contained 6, 1, 2 , 4, 4, 4 and 1 genotypes, respectively. These results revealed that marker AP3206f would be best in screening 22 rice genotypes followed by RM1287, RM7075, and RM10793 according to PIC values. These findings can have the potential role for further improvement of salinity tolerance rice genotypes through marker-assisted breeding.
\end{abstract}

Keywords: diversity; marker; PIC; salt tolerance; SSR; UPGMA

\section{Introduction}

Rice is the world's third most important cereal crop growing on over 161.1 million hectares of land which provides 487.5 metric tons milled rice globally (Statista, 2019). Rice is the dominant food crop of around 158.9 million Bangladeshi people (BBS, 2017). With the ever-increasing population and unfavorable effects of climate change, it becomes a burning issue to increase the current production of rice. Bangladesh has already become one of the potential victims of climate change. Salt stress is one of the most important abiotic stresses that influences rice production by altering the metabolism, reducing water potential, through ionic imbalances and toxicity thus ultimately reduces plant growth and developments (Joseph and Jini, 2011; Tuteja et al., 2012). Rice is very sensitive to salt stress and is currently listed as the most salt sensitive cereal crop with a threshold 
of $3 \mathrm{dSm}^{-1}$ for most cultivated varieties (USDA, 2013). However, there exists tremendous variation for salt tolerance within the species of rice (Sabouri et al., 2009) which provides various opportunities improving salt stress tolerance in rice by genetic means. Progress in salt tolerance breeding is slow due to limited knowledge in the genetics of tolerance, complexity of the several tolerance mechanisms involved, inadequate screening techniques, low selection efficiency and poor understanding of salinity and environmental interactions (Bhowmik et al., 2009). Thus, breeding for enhancing tolerances to salt stress in rice is the major objective for the development of varieties for saline prone ecologies. Actually, in breeding or any crop improvement program, genetic diversity has a great role to play for safeguarding competent use and also for choosing efficient breeding strategies (Chakravarthi and Naravaneni, 2006). In plants, different morphological or physiological characters including isozyme or protein markers are more frequently utilized for determining genetic diversity. However, these markers have some disadvantages due to environmental influence, long assessment time and low polymorphism between the genotypes (Chakravarthi and Naravaneni, 2006). For these reasons, use of molecular markers has evolved as more distinct, consistent, and proficient genetic diversity tool for germplasm characterization, conservation and management (Chakravarthi and Naravaneni, 2006). Among the molecular markers, microsatellites or SSRs (simple sequence repeats) are hugely chosen as efficient tool in genetic diversity study due to their high polymorphism, quickness, simplicity, accuracy, and transferability (Ma et al., 2011; Roy et al., 2015). Conventional breeding is accelerated by the use of molecular markers linked to the tolerance trait being intro-grassed, which is known as MAS (marker-assisted selection). This allelic variation in the gene of interest can provide both a novel source of genetic material for MAS and can also be used to design an easy, cheap molecular marker, thus providing a perfect marker for a trait contributing to stress tolerance like salt tolerance. There are about 15,000 SSRs marker now available for rice (www.gramene.org) and currently being used to develop high density genetic maps, genotype rice accessions, genetic structure and diversity patterns determination, optimization of the assembly of core collections, and for marker-assisted breeding (McCouch, 2002; Yu et al., 2003; Garris et al., 2005). Therefore, in present study, twenty-two advanced rice lines were analyzed to study the genetic diversity and relationships among them using SSR markers.

\section{Materials and Methods}

\subsection{Plant material}

The study was conducted at the experimental farm of Bangladesh Institute of Nuclear Agriculture (BINA), Mymensingh, during January 2013 to August 2014. Twenty-two rice lines with diverse genetic background which were collected from different sources were used in this study (Table 1).

\subsection{Collection of Leaf Samples and DNA Extraction}

Juvenile, vigorously growing fresh leaf samples was collected from 21-30 days old seedlings for isolation of genomic DNA. At first, the healthy portion of the youngest leaves of the tiller was cut apart with sterilized scissors and washed in ethanol (70\%) and distilled water. Genomic DNA samples of each genotype were extracted from vigorous, young growing leaf tissues using the mini preparation Modified Cetyl Trimethyl Ammonium Bromide (CTAB) method (IRRI, 1997).

\subsection{Primer selection and DNA amplification}

A total of four SSR markers were screened (Table 2) to yield amplification products on the total DNA obtained from the leaf tissues. The following PCR materials were used for PCR: $1.0 \mathrm{ml} 10 \mathrm{x}$ PCR buffer, $0.2 \mathrm{ml}$ Taq DNA polymerase, $1.0 \mathrm{ml}$ forward primer, $1.0 \mathrm{ml}$ reverse primer, $1.0 \mathrm{ml}$ dNTPs, $3.8 \mathrm{ml}$ sterilized $\mathrm{dH}_{2} \mathrm{O}$ and $2.0 \mathrm{ml}$ template DNA for total volume of $10 \mathrm{ml}$. For amplification, the thermal cycler was set at 1 cycle for $5 \mathrm{~min}$ at $94^{\circ} \mathrm{C}$ as an initial hot start and strand separation step. This was followed by 2 nd program having 35 cycles, which comprises denaturation $\left(94^{\circ} \mathrm{C}\right)$ for $1 \mathrm{~min}$, annealing $\left(55^{\circ} \mathrm{C}\right)$ for $1 \mathrm{~min}$ and primer elongation $\left(72^{\circ} \mathrm{C}\right)$ for from $2 \mathrm{~min}$. Finally, 1 cycle of $7 \mathrm{~min}$ at $72^{\circ} \mathrm{C}$ was used for final extension and amplified products were stored at $-20^{\circ} \mathrm{C}$ until further use. The amplification products were separated on $8 \%$ polyacrylamide gel in $0.5 \mathrm{x}$ TBE buffer. The DNA band patterns were visualized under UV light and photographed using a polaroid camera. 


\subsection{Analysis of SSR Data}

The size (in nucleotide base pairs) of the amplified band for each microsatellite marker was determined based on its migration relative to a molecular weight size marker ( 25 bp DNA Ladder) with the help of Alpha Viewer software.

The summary statistics including the number of alleles per locus, major allele frequency, gene diversity and Polymorphism Information Content (PIC) values were determined using Power Marker version 3.23 (Liu and Muse, 2005), a genetic analysis software. Molecular weights for microsatellite products, in base-pairs, were estimated with Alpha viewer software. The individual fragments were assigned as alleles of the appropriate microsatellite loci. Polymorphism Information Content (PIC) value described by Anderson (1993) for selfpollinated species were calculated as follows:

$\mathrm{PICi}=1-\sum_{\mathrm{i}=1}^{\mathrm{n}}(\mathrm{Pij})^{2}$

Where, Pij is the frequency of the $\mathrm{jth}$ allele for ith marker and summed over $\mathrm{n}$ alleles.

\section{Results}

The microsatellite enriched DNA fingerprint were constructed using the standard procedures. In this study, 22 lines of rice were analyzed using four primers. Amplified microsatellite loci were analyzed for polymorphism using Polyacrylamide Gel Electrophoresis (PAGE) and the result revealed that all the primers detected polymorphism among the rice lines analyzed (Figure 1). The microsatellite loci were also polymorphic (3 to 5) alleles per locus with a mean of 4.25 alleles per locus in the present study. The bands obtained from other lines were compared to the band obtained from salt tolerant variety like Binadhan-8, Binadhan-10, FL- 478 (Figure 1). In respect of primer AP3206f, allele size ranged from 154bp-164bp, whereas primer RM1287, RM7075, RM10793, showed a range 163 bp-175bp, 149bp to 159 bp, 113 bp to 126bp (Table 3) respectively. Using Four SSR markers, a total of 25 alleles were detected among the 22 rice lines. The average number of alleles per locus was 4.25, with a range of 3 (RM7075) to 5 (AP3206f \& RM1287) (Table 4). An allele observed in less than $5 \%$ of the 22 accessions was considered to be rare. Rare alleles were observed at all of the SSR loci with an average of 1.25 rare alleles per locus (Table 4). In general, markers detecting a greater number of alleles per locus detected more rare alleles. Marker RM 1287 detected the highest number of alleles (5) and rare alleles (2) (Table 4). Major allele is defined as the allele with the highest frequency and also known as most common allele at each locus. The size of the different major alleles at different loci ranges from 113bp (RM10793) to 163bp (RM1287) (Table 5). On average, $46.59 \%$ of the 22 rice lines shared a common major allele ranging from $36.36 \%$ (AP3206f) to $50 \%$ (RM10793) common allele at each locus (Table 5).

The highest gene diversity (0.7810) was observed in loci AP3206f and the lowest gene diversity (0.5620) was observed in loci RM7075 with a mean diversity of 0.6663 (Table 4). It was observed that marker detecting the lower number of alleles showed lower gene diversity than those which detected higher number of alleles which revealed higher gene diversity. Polymorphism information content (PIC) value is a reflection of allele diversity and frequency among the varieties. PIC value of each marker can be evaluated on the basis of its alleles. PIC varied significantly for all the studied SSR loci. In the present study, the level of polymorphism among the 22 rice lines was evaluated by calculating PIC values for each of the 4 SSR loci. The PIC values ranged from 0.4762 (RM7075) to 0.7524 (AP3206f) with an average of 0.61 per locus (Table 5).

The UPGMA-based dendrogram was constructed based on the binary data obtained from the SSR marker-based DNA profiles of the sample analyzed (Figure 2). The correlation coefficient value used to construct the dendrogram was 0.58 . The genotypes with genetic similarity clustered together in the dendrogram and we observed seven major clusters where cluster I contained most of the genotypes. Cluster I, II, III, IV, V, VI, and VII contained 6, 1, 2, 4, 4, 4 and 1 genotypes, respectively (Figure 2). Among the total genotypes those which showed comparatively same banding patterns were grouped into the same cluster. 
Table 1. List of plant materials used in the study.

\begin{tabular}{lll}
\hline Sl no. & Lines & Source \\
\hline G1 & RC 191 & IRRI* \\
G2 & RC 192 & IRRI* \\
G3 & RC 193 & IRRI* \\
G4 & RC 217 & IRRI* \\
G5 & RC 221 & IRRI* \\
G6 & RC 222 & IRRI* \\
G7 & RC 225 & IRRI* \\
G8 & RC 227 & IRRI* \\
G9 & RC 229 & IRRI* \\
G10 & RC 251 & IRRI* \\
G11 & RC 249 & IRRI* \\
G12 & BRRI dhan11 & BRRI \\
G13 & Binadhan 7 & BINA \\
G14 & Binadhan 8 & BINA \\
G15 & Binadhan 10 & BINA \\
G16 & Binadhan 11 & BINA \\
G17 & Cherang & BINA \\
G18 & Binadhan 12 & BINA \\
G19 & BRRI dhan29 & BRRI \\
G20 & Pajam & BRRI \\
G21 & BRRI dhan39 & BRRI \\
G22 & FL 478 & BRRI \\
\hline
\end{tabular}

Table 2. Information of SSR markers used for the study.

\begin{tabular}{|c|c|c|c|}
\hline Primer & Expected product size (bp) & Primer Sequence (5'-3') & Annealing temp. $\left({ }^{\circ} \mathrm{C}\right)$ \\
\hline AP3206f & 167 & $\begin{array}{l}\text { Fw: GCAAGAATTAATCCATGTGAAAGA } \\
\text { Rv: ATGCTCTGGCTCCCT CAAG }\end{array}$ & 55 \\
\hline RM1287 & 162 & $\begin{array}{l}\text { Fw: GGAAGCATCATGCAATAGCC } \\
\text { Rv: GGCCGTAGTTTTGCTACTGC }\end{array}$ & 55 \\
\hline RM7075 & 155 & $\begin{array}{l}\text { Fw: TATGGACTGGAGCAAACCTC } \\
\text { Rv: GGCACAGCACCAATGTCTC }\end{array}$ & 55 \\
\hline RM10793 & 124 & $\begin{array}{l}\text { Fw: GACTTGCCAACTCCTTCAATTCG } \\
\text { Rv: TCGTCGAGTAGCTTCCCTCTCTACC }\end{array}$ & 55 \\
\hline
\end{tabular}

Table 3. Size of alleles at 4 SSR loci of 22 rice lines.

\begin{tabular}{lll}
\hline SSR markers & Allele Size (bp) & Allele frequency \\
\hline & 6 & 0.1364 \\
AP3206f & 16 & 0.3636 \\
& 4 & 0.0909 \\
& 4 & 0.0909 \\
& 8 & 0.1818 \\
& 6 & 0.1364 \\
\hline \multirow{2}{*}{ RM1287 } & 4 & 0.0909 \\
& 20 & 0.4545 \\
& 10 & 0.2273 \\
& 6 & 0.1364 \\
RM7075 & 2 & 0.0455 \\
& 2 & 0.0455 \\
\hline & 4 & 0.0909 \\
RM10793 & 24 & 0.5455 \\
& 16 & 0.3636 \\
\hline & 16 & 0.3636 \\
& 22 & 0.5000 \\
& 2 & 0.0455 \\
& 2 & 0.0455 \\
& & 0.0455 \\
\hline
\end{tabular}


Table 4. Summary information on 4 SSR markers used in the present study.

\begin{tabular}{lllllll}
\hline Marker & Chromo. location & No. of Allele found & Frequency & *Rare alleles & Size range & Gene diversity \\
\hline AP3206f & 1 & 5 & 0.1727 & 0 & $154-164$ & 0.7810 \\
RM1287 & 1 & 5 & 0.1818 & 2 & $163-175$ & 0.7107 \\
RM7075 & 1 & 3 & 0.3333 & 0 & $149-159$ & 0.5620 \\
RM10793 & 1 & 4 & 0.1591 & 3 & $113-126$ & 0.6116 \\
\hline Mean & & $\mathbf{4 . 2 5}$ & $\mathbf{0 . 2 1 1 7 2 5}$ & $\mathbf{1 . 2 5}$ & & $\mathbf{0 . 6 6 6 3 2 5}$ \\
\hline
\end{tabular}

Table 5. Data on Major alleles (size and Frequencies) and Polymorphism information Content (PIC) found among 22 rice lines for 4 microsatellites (SSR) marker.

\begin{tabular}{|c|c|c|c|}
\hline \multirow[b]{2}{*}{ Locus name } & \multicolumn{2}{|c|}{ Major allele } & \multirow[b]{2}{*}{ PIC } \\
\hline & Size (bp) & Frequency $(\%)$ & \\
\hline AP3206f & 154 & 36.36 & 0.7524 \\
\hline RM1287 & 163 & 45.45 & 0.6729 \\
\hline RM7075 & 151 & 54.55 & 0.4762 \\
\hline RM10793 & 113 & 50.00 & 0.5407 \\
\hline Mean & 145.25 & 46.59 & 0.61055 \\
\hline
\end{tabular}

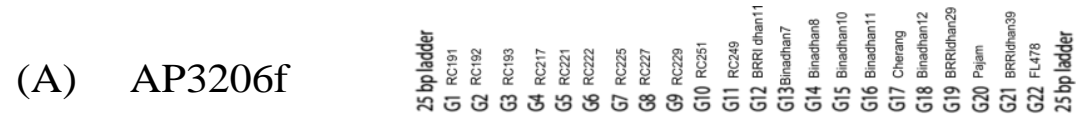

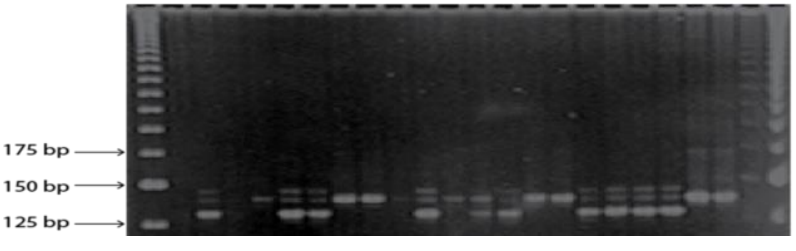

(B) RM1287
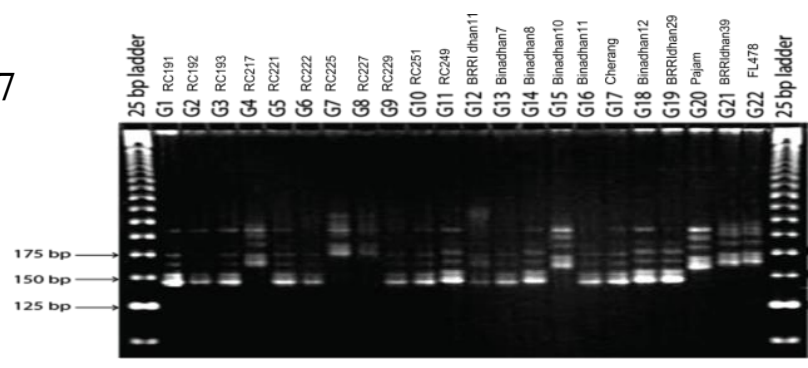

(C) RM7075
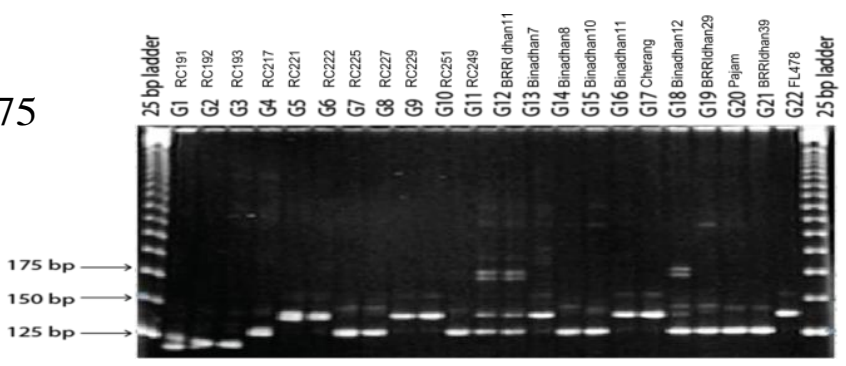

(D) RM10793
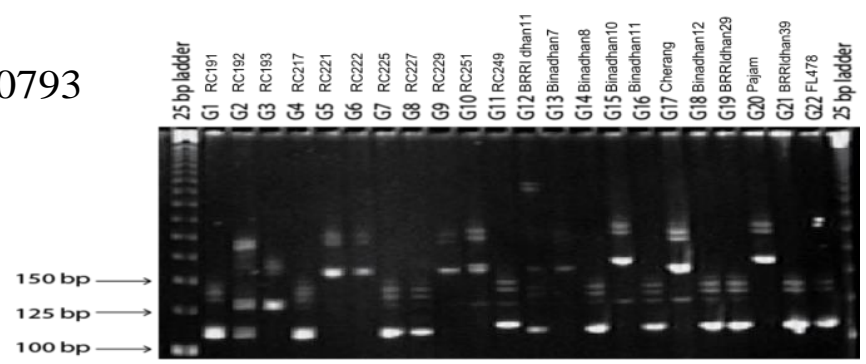

Figure 1. SSR profile of 22 rice genotypes using different marker AP3206f (A), RM1287 (B), RM7075 (C) and RM10793 (D). 


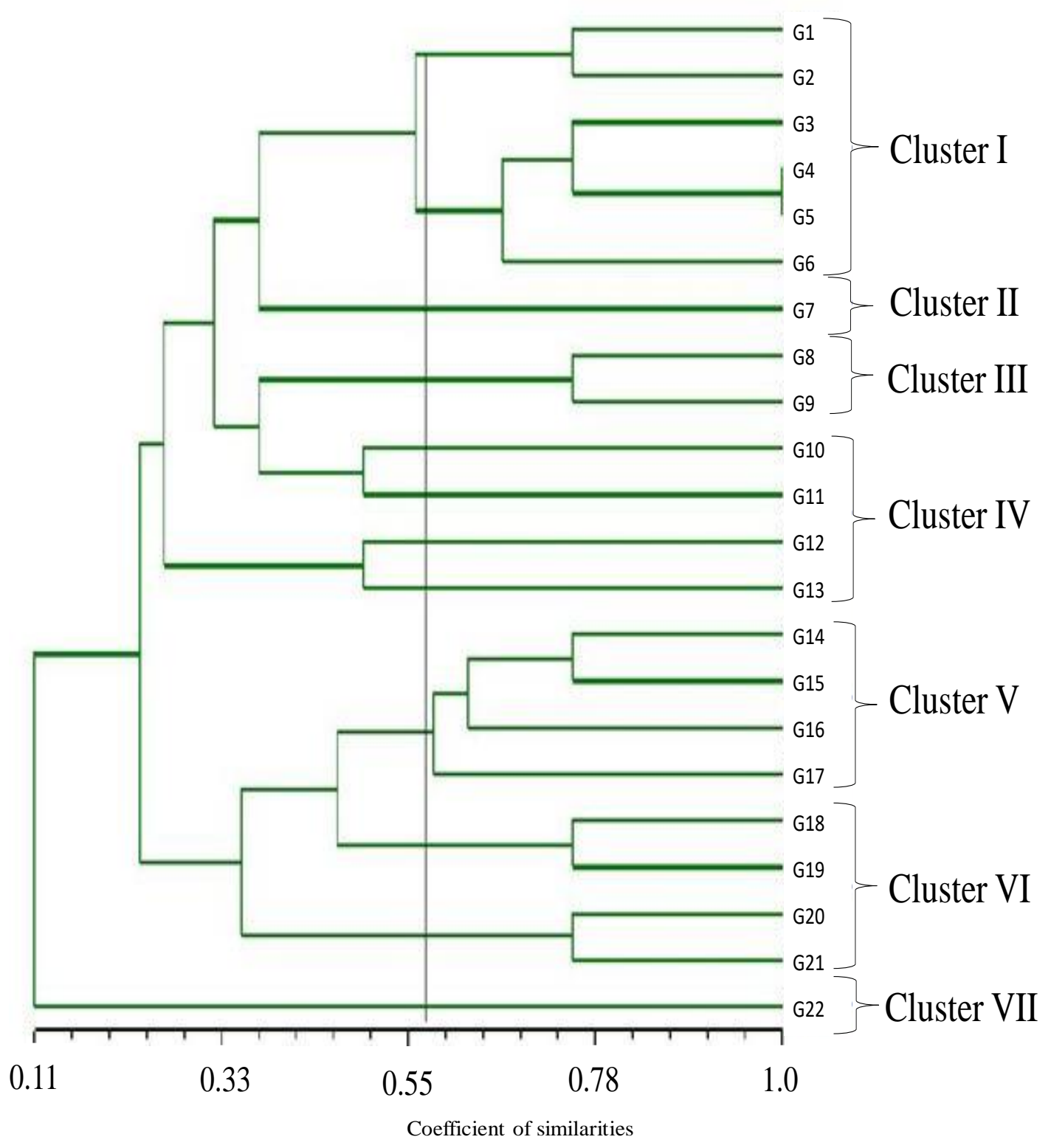

Figure 2. UPGMA dendrogram showing the genetic relationship among 22 rice genotypes.

\section{Discussion}

Using 4 SSR markers, a total of 17 alleles were detected among the 22 rice lines. The average number of alleles per locus was 4.25, with a range of 3 (RM7075) to 5 (AP3206f \& RM1287). These values were comparable to those reported earlier (4.06 alleles per locus; range 2 to 9) by Ahmed et al. (2019) who used 16 SSR primers to estimate genetic relationship among 30 rice genotypes. An allele observed in less than $5 \%$ of the 22 accessions was considered to be rare allele (Zhu et al., 2011). Rare alleles were observed at all of the SSR loci with an average of 1.25 rare alleles per locus. In general, markers detecting a greater number of alleles per locus detected more rare alleles. Marker RM1287 detected the highest number of alleles (5) and rare alleles (2). According to Jain et al. (2004) rare alleles are highly informative in fingerprinting of rice varieties. The size of the different major alleles at different loci ranges from 113bp (RM10793) to 163 bp (RM1287) (Table 4). Similar result was reported by Ahmed et al. (2019) where the size of the different major alleles at different loci ranges from 100bp (RM277) to $312 \mathrm{bp}$ (RM594). On average, $46.59 \%$ of the 22 rice lines shared a common major allele ranging from $36.36 \%$ (AP3206f) to $50 \%$ (RM10793) common allele at each locus. Rana et al. (2018) reported that on average, $40 \%$ of the 10 rice genotypes shared a common major allele ranging from $30 \%$ (RM351) to 50\% (RM510) common allele at each locus.

Gene diversity is the most widely used parameter to estimate genetic variability within the genotypes (Nei, 1973; Toro and Caballero, 2005). Herrera et al. (2008) and Rana et al. (2018) opined that the marker detecting 
the lower number of alleles showed lower gene diversity than those detected higher number of alleles which revealed higher gene diversity. The highest gene diversity (0.7810) was observed in loci AP3206f and the lowest gene diversity (0.5620) was observed in loci RM7075 with a mean diversity of 0.6663 (Table 4). The results from our study agree well with the average gene diversity of 0.59 (range of 0.27 to 0.87 ) by Pathaichindachote et al. (2019). Polymorphism information content (PIC) value is a reflection of allele diversity and frequency among the varieties (Rashid et al., 2018). PIC value of each marker can be evaluated on the basis of its alleles. PIC varied significantly for all the studied SSR loci. In the present study, the level of polymorphism among the 22 rice lines was evaluated by calculating PIC values for each of the 4 SSR loci. The PIC values ranged from 0.4762 (RM7075) to 0.7524 (AP3206f) with an average of 0.61 per locus (Table 4). The highest PIC value was 0.7524 for AP3206f. The lowest PIC value observed 0.4762 for RM7075. The findings is in line with the reported PIC value in previous work by Islam et al. (2018) who stated that the values of Polymorphic information content (PIC) ranged from 0.217 to 0.835 with an average of 0.495 . This result revealed that markers AP3206f would be best in screening 22 rice lines followed by RM1287, RM7075 and RM10793. Almost similar results were obtained by Vabna et al. (2021) where RM206 marker showed a PIC of 0.49 and considered as the best marker among the studied markers for 24 rice landraces. Genetic similarities were calculated from the data of Nei's coefficient (1973). The similarly matrix was used to determine the level of relatedness among the studied lines. Pair-wise estimates of similarity ranged from 0 to 1.00 and the average similarity among all 22 cultivars was 0.58 . The lowest genetic distance (0) was observed in G1 Vs G2, G10 vs G17, G11 vs G14, G12 vs G18, G13 vs G17, and G19 vs G17. The highest genetic distance of 1 was observed between a number of accession or variety pair. Some of them G1 vs G11, G10 vs G22, G16 vs G11, G5 vs G18, G21 vs G13 etc. These results corroborate with the findings of Ahmed et al. (2019) who observed genetic distance between rice genotypes for the three primers ranging from 0.88 to 0.36 . UPGMA method was used for cluster analysis to differentiate the studied lines into groups based on similarity coefficient. Twelve clusters were made at genetic similarity level of 0.11 to 0.58 . All of the 22 rice lines were grouped in seven clusters. This result is consistent with previous work done by Rana et al. (2018) who also grouped 10 genotypes with SSR markers into 4 clusters based on UPGMA method. In addition, Tabkhkar et al. (2012) also grouped 48 rice lines with SSR markers into four main clusters.

Finally, it can be concluded that the use of microsatellite markers divulged the larger range of similarity values for lines. Microsatellite markers offer a great opportunity for the assessments of genetic diversity and relationships, which can be helpful in future breeding programs. By using the data from clustering, intercrossing may be possible between distant rice lines.

\section{Conclusions}

From the analysis, it was found that a total of 12 alleles were detected with an average number of 4.25 alleles per locus. The PIC values ranged from 0.4762 to 0.7524 with an average of 0.611 . AP3206f was the best marker for identification of lines as revealed by PIC values. The size of the different major alleles at different loci ranges from 113bp (RM10793) to 163bp (RM1287). According to Nei (1973), the highest gene diversity (0.7810) was observed in loci AP3206f and the lowest gene diversity (0.5620) was observed in loci RM7075 with a mean diversity of 0.667 . The genotypes with genetic similarity clustered together in the dendrogram based on UPGMA method and we observed seven major clusters. Our study revealed that markers AP3206f would be best in screening 22 rice lines followed by RM1287, RM7075 and RM10793. SSR markers used in this study were convenient, polymorphic and associated with salinity tolerance.

\section{Acknowledgements}

Special thanks to the authority of BAU and BINA (specially Biotechnology Division) for giving me such kind off research opportunities.

\section{Conflict of interest}

None to declare.

\section{References}

Ahmed MS, TR Anik, MA Islam, MI Uddin and MS Haque, 2019. Screening of some rice (Oryza sativa L.) genotypes for salinity tolerance using morphological and molecular markers. Biosci. Biotechnol. Res. Asia, 16: 377-390.

Anderson JA, ME Sorrells and SD Tanksley, 1993. RFLP analysis of genomic regions associated with resistance to pre-harvest sprouting in wheat (Triticum aestivum). Crop Sci., 33: 453-459. 
BBS, 2017. Yearbook of Agricultural Statistics. Bangladesh Bureau of Statistics, Statistics and Informatics Division (SID), Ministry of Planning, Government of the People's Republic of Bangladesh, Dhaka. p. 39.

Bhowmik SK, S Titov, MM Islam, A Siddika, S Sultana and MDS Haque, 2009. Phenotypic and genotypic screening of rice genotypes at seedling stage for salt tolerance. Afr. J. Biotechnol., 8: 6490-6494.

Chakravarthi BK and R Naravaneni, 2006. SSRmarker basedDNA fingerprinting and diversity study in rice (Oryza sativa L). Afr. J. Biotechnol., 5: 684-688.

Garris AJ, TH Tai, J Coburn, S Kresovich and S McCouch, 2005. Genetic structure and diversity in Oryza sativa L. Genetics, 169: 1631-1638.

Herrera G, PD Dunia, PA Iris, TN Gelis, P Alejandro, PM Cesar and MT Joe, 2008. Assessment of genetic diversity invenezuelan rice cultivars using simple sequence repeats markers. Electron. J. Biotechnol., 11: 5.

IRRI, 1997. International Rice Research Institute. Annual Report for 1967. Los Baños, Laguna, Philippines. p. 308.

Islam MA, TR Anik, MM Hossain, MI Uddin and MS Ahmed, 2018. Genetic diversity analysis of some Bangladeshi aromatic rice (Oryza sativa L.) using simple sequence repeat markers (SSRM). Arch. Agric. Environ. Sci., 3: 297-303.

Jain S, RK Jain and SR McCouch, 2004. Genetic analysis of Indian aromatic and quality rice (Oryza sativa L.) germplasm using panels of fluorescently-labeled microsatellite markers. Theor. Appl. Genet, 109: 965-977.

Joseph B, D Jini and S Sujatha, 2010. Biological and physiological perspectives of specificity in abiotic salt stress response from various rice plants. Asian J. Agric. Sci., 2: 99-105.

Liu K and SV Muse, 2005. PowerMarker: an integrated analysis environment for genetic marker analysis. Bioinformatics, 21: 2128-2129.

Ma H, Y Yin, ZF Guo, LJ Chen, L Zhang, M Zhong and GJ Shao, 2011. Establishment of DNA fingerprinting of Liaojing series of japonica rice. Middle East J. Sci. Res., 8: 384-392.

McCouch SR, 2002. Development and mapping of 2240 new SSR arkers for rice (Oryza sativa L.). DNA Res., 9: 199-207.

Nei M, 1973. Analysis of gene diversity in subdivided populations. Proc. Natl. Acad. Sci. U. S. A., 70: 33213323.

Pathaichindachote W, N Panyawut, K Sikaewtung, S Patarapuwadol and A Muangprom, 2019. Genetic diversity and allelic frequency of selected Thai and exotic rice germplasm using SSR markers. Rice Sci., 26: 393-403.

Rana MM, MA Islam, S Imran, S Rubani and L Hassan, 2018. Genetic diversity analysis of NERICA lines and parents using SSR markers. Intl. J. Plant Soil Sci., 23: 1-10.

Rashid MM, S Imran, MA Islam and L Hassan, 2018. Genetic diversity analysis of rice landraces (Oryza sativa L.) for salt tolerance using SSR markers in Bangladesh. Fundam. appl. agric., 3: 460-466.

Roy S, A Banerjee, B Mawkhlieng, AK Misra, A Pattanayak and GD Harish, 2015. Genetic diversity and population structure in aromatic and quality rice (Oryza sativa L.) landraces from North Eastern India. PLoS One, 10: e0129607.

Sabouri H and A Biabani, 2009. Toward the mapping of agronomic characters on a rice genetic map: Quantitative Trait Loci analysis under saline condition. Biotechnology, 8: 144-149.

Statista, 2019. Grain Production Worldwide by Type, 2017/2018. Cited July 7, 2018.

Tabkhkar N, B Rabiei and A Sabouri, 2012. Genetic diversity of rice cultivars by microsatellite markers tightly linked to cooking and eating quality. Aust. J. Crop Sci., 6: 980-985.

Toro MA and A Caballero, 2005. Characterization and conservation of genetic diversity in subdivided populations. Philos. Trans. R. Soc. Lond. B, Biol. Sci., 360: 1367-1378.

Tuteja N, LP Singh, SS Gill, R Gill and R Tuteja, 2012. Salinity Stress: A Major Constraint in Crop Production. In: Improving Crop Resistance to Abiotic Stress. Wiley-VCH Verlag GmbH \& Co. KGaA., pp. 71-96. DOI: 10.1002/9783527632930.ch4.

USDA, 2013. Bibliography on Salt Tolerance. Fibres, Grains and Special Crops. Riverside, CA: George E. Brown, Jr. Salinity Lab.US Department Agriculture, Agriculture Research Service.

Vabna FA, MZ Islam, MFRK Prince and ME Hoque, 2021. Molecular diversity analysis in Boro Rice (Oryza sativa L.) landraces using SSR markers. Asian J. Biol., 12: 36-48.

Yu SB, WJ Xu, CHM Vijayakumar, J Ali, BY Fu, JL Xu, YZ Jiang, R Marghirang, J Domingo, C Aquino, SS Virmani and Li ZK, 2003. Molecular diversity and multilocus organization of the parental lines used in the international rice molecular breeding program. Theor. Appl. Genet., 108:131-140.

Zhu C, X Li and J Yu, 2011. Integrating Rare-Variant Testing, Function Prediction, and Gene Network in Composite Resequencing-Based Genome-Wide Association Studies (CR-GWAS). G3-GENES GENOM GENET., 1: 233-243. 